\title{
Survivorship care planning in gynecologic oncology-perspectives from patients, caregivers, and health care providers
}

\author{
Belle H. de Rooij ${ }^{1,2,3}$ (D) Teresa Hagan Thomas ${ }^{4} \cdot$ Kathryn E. Post $^{1,5} \cdot$ Jane Flanagan $^{1,5} \cdot$ Nicole P. M. Ezendam ${ }^{1,2}$. \\ Jeffrey Peppercorn ${ }^{1} \cdot$ Don S. Dizon ${ }^{6}$
}

Received: 6 April 2018 / Accepted: 28 August 2018 / Published online: 12 September 2018

(C) The Author(s) 2018

\begin{abstract}
Purpose This qualitative study sought to describe the challenges following treatment and the preferences regarding survivorship care among patients treated for gynecological cancer, their caregivers, and health care providers.

Methods Between July and August 2017, in-depth semi-structured interviews regarding survivorship were conducted at a large academic hospital in the USA among patients who recently completed treatment $(<12$ months) for a gynecological cancer (ovarian, endometrial, cervical, and vulvar) and their primary caregivers. A focus group was conducted among health care providers (oncologists, nurses, and fellows). Main themes were identified using descriptive content analysis.

Results A total of 30 individuals participated in this study (13 patients, 9 caregivers, 8 health care providers). Almost all participants reported a desire for more information on how to address survivorship needs, specifically as they related to side effects, follow-up schedule, and psychological assistance. Despite this uniformly identified need for more information, preferences for survivorship care planning differed across cancer types and individuals, with respect to content, timing, and mode of delivery. Health care providers expressed challenges in communicating with patients about survivorship, a desire to shift posttreatment conversations to the goal of improving quality of life as opposed to focusing on disease recurrence, and an unmet need for disease specific and individualized survivorship care planning.

Conclusions Patients, caregivers, and health care providers each expressed a need for gynecologic cancer-tailored survivorship care resources.

Implications for Cancer Survivors The variation of disease types and patient and caregiver needs may require multi-faceted, individualized survivorship care planning.
\end{abstract}

Keywords Cancer survivorship · Survivorship care · Survivorship care plan · Gynecologic cancer · Qualitative research

Belle H. de Rooij and Teresa Hagan Thomas contributed equally to this work.

Electronic supplementary material The online version of this article (https://doi.org/10.1007/s11764-018-0713-9) contains supplementary material, which is available to authorized users.

Belle H. de Rooij

b.h.derooij@uvt.nl

1 Massachusetts General Hospital Cancer Center, Harvard Medical School, Boston, MA, USA

2 CoRPS - Center of Research on Psychology in Somatic diseases, Department of Medical and Clinical Psychology, Tilburg University, Tilburg, The Netherlands
3 The Netherlands Comprehensive Cancer Organisation, Utrecht, The Netherlands

4 University of Pittsburgh School of Nursing, Pittsburgh, PA, USA

5 Boston College William F. Connell School of Nursing, Boston, MA, USA

6 Lifespan Cancer Institute/Rhode Island Hospital, Alpert Medical School of Brown University, Providence, RI, USA 


\section{Introduction}

Each year, almost 106,000 women in the USA are diagnosed with a gynecological cancer [1]. Ovarian cancer remains the deadliest gynecological cancer, followed by vaginal, cervical, endometrial, and vulvar cancers. The estimated 5 -year survival rates vary widely from $46 \%$ for women with ovarian cancer to over $80 \%$ for women with endometrial or vulvar malignancies [1]. Survival rates for gynecological cancers have slightly increased during the past decades, reflecting advances in treatment that ultimately help patients survive their disease [1].

Regardless of prognosis, a wide body of literature supports the notion that women treated for gynecological cancer experience a significant level of disease- and treatment-related symptoms that persist after the end of first-line treatment and greatly impact their long-term quality of life [2-7]. In addition, one of the most significant and overwhelming psychological concerns cancer survivors struggle to manage is the fear of cancer recurrence, which is associated with increased symptom burden, overwhelming anxiety, post-traumatic stress, and hopelessness $[4,6,8-10]$.

The National Academy of Medicine (NAM) acknowledged that these factors are important in the ongoing care for cancer patients ending treatment, and in 2006 recommended that all cancer survivors receive a Survivorship Care Plan (SCP) [11]. SCPs typically contain written information on diagnosis, documentation of all treatments, short- and long term effects of the treatments, and recommendation for follow-up care [11]. To date, multiple randomized controlled trials evaluating the impact of SCPs among cancer patients [12-17], including gynecological cancer [13, 16, 18], have failed to demonstrate beneficial effects of SCPs on short- or long-term satisfaction with information provision and care, quality of life, or distress. These outcomes suggest that patients with gynecological cancer may not receive the intended benefits of an SCP as proposed by the NAM without further refinement and evaluation of these interventions [19].

Part of the disconnect between the prominent calls for SCPs as a self-evident beneficial intervention and the lack on strong evidence supporting SCPs in their current format may stem from a failure to adequately tailor these interventions to the needs of specific cancer patient populations. Additionally, there may be a need to further adapt the intervention to the needs and preferences of the individual patient. Given the lack of evidence to support existing SCP models in the setting of gynecologic oncology, we sought to describe the perspectives of patients with gynecological cancer, as well as their caregivers and health care providers (HCPs). This may provide insights into the unmet needs of patients and their caregivers as well as challenges to the health care team and identify opportunities for effective intervention through SCPs or other aspects of survivorship care.
The aim of the current study is to describe the (1) challenges following treatment and (2) the preferences regarding survivorship care among patients treated for gynecological cancer, their caregivers, and HCPs.

\section{Methods}

\section{Design}

This study employed an open-ended qualitative descriptive design including in-depth semi-structured interviews among three stakeholder groups: patients with a gynecological cancer, their caregivers, and gynecologic oncology HCPs with whom we conducted a focus group. The study protocol was approved by the Dana-Farber/Harvard Cancer Center Institutional Review Board.

\section{Participants and recruitment}

Patients older than 18, able to read and respond in English, that completed treatment for any type of gynecological cancer within the past 12 months, were eligible to participate in the study. The study team reviewed upcoming clinic schedules for eligible patients and invited those patients to participate during a scheduled outpatient clinic visit at the Cancer Center, or were invited by phone. Patients were asked to identify their primary caregiver defined as a spouse, family member, or friend who provides care and support to the patient. Caregivers were introduced to the study at the Cancer Center, or by phone when not present at the Cancer Center. After informed consent, telephone or in-person interviews with patients and caregivers were scheduled at a time convenient for them. HCPs that primarily provide care for patients with gynecologic cancer (gynecologic oncolgists, medical oncologists, gynecologic oncology fellows, and nurse practitioners) were invited to participate in a focus group interview during a regular gynecologic oncology staff meeting. Informed consent was obtained at the beginning of the meeting.

\section{Data collection}

Telephone or in-person interviews with patients and caregivers and the HCP focus group were digitally recorded. Audio-recordings were transcribed verbatim to text using TranscribeMe.com. In addition, demographic questionnaires were completed by patients and caregivers. Measures included age, sex, ethnicity, education, and employment. Clinical patient data was extracted from each patient's electronic medical record. 


\section{Interview guide}

Semi-structured qualitative interview guides were developed by members of the study team. We purposefully included open-ended questions to determine patient and caregiver needs a priori with minimal predetermined categories of survivorship care planning topics. While the patient and caregiver questionnaire guides included similar items tailored for each group, separate questionnaire guides were developed for HCPs. The interview guides were discussed and refined by study team members resulting in a list of questions and follow-up probes for each group.

\section{Data analysis}

Data transcripts were coded in NVivo 11 (QSR International) using descriptive content analysis techniques. The initial coding scheme for patient and caregiver interviews was based on the first three patient interviews and iteratively refined and expanded while reviewing additional interviews. Two study researchers (THT and BHR) generated the categories independently through a close reading of the transcript texts, jointly comparing their categories, reviewing any discrepancies and disagreements, and resolving discrepancies through consensus. We continued reviewing coding until saturation was achieved and no new category themes emerged. After developing a comprehensive list of categories, we then summarized and classified the categories into higher-order themes. To ensure consistency between themes, categories, and the raw data, we selected representative quotations of each theme to illustrate its meaning and assist with data interpretation. Codebooks were developed for patient interviews first and applied to the caregivers' interviews after determining similar content between these interviews. The HCP focus group was coded separately due to their distinct perspective and ideas discussed. We calculated the frequency of specific categories and compare these to patients' and caregivers' responses. Based on emerging categories indicating differences in type of gynecological cancer, we also decided to compare the perspectives of patients and caregivers with ovarian cancer versus other gynecological cancer types. As a qualitative study, we focused our comparisons on basic descriptive statistics and did not use our quantified data to statistically test group differences to avoid over-simplifying our qualitative exploratory data.

\section{Results}

In total, we had 30 participants included in this study (13 patients, 9 caregivers, and $8 \mathrm{HCPs})$. Five patients that were approached did not want to participate (no time/busy or did not want to be reminded of their cancer) and one patient was lost to follow-up. Four caregivers identified by patients chose not to participate in the study. Only one patient had an inperson interview, and all other patients and caregivers preferred telephone interviews. Interviews for patient and caregiver participants lasted 30-40 min. The focus group interview of the providers was $35 \mathrm{~min}$.

\section{Participant characteristics}

Table 1 describes patient and caregiver characteristics. Patients $(n=13)$ represented various gynecological cancer types, including ovarian $(n=5)$, endometrial $(n=4)$, cervical $(n=2)$, fallopian tube $(n=1)$, and vulvar $(n=1)$, had an average age of 63 , were predominantly white $(92 \%)$, unemployed at the time of the interview (62\%), and completed treatment 6 months before the interview. Caregivers of patients $(n=9)$ were mostly the patient's spouse $(n=6)$, had an average age of 59 , were predominantly male $(78 \%)$, white $(100 \%)$, and employed at the time of the interview (56\%). The HCP focus group $(n=8)$ included gynecologic oncolgists $(n=2)$, gynecologic oncology fellows $(n=3)$, a medical oncologist $(n=1)$, a radiation oncologist $(n=1)$, and a nurse practitioner $(n=1)$, and were predominantly female $(n=5,63 \%)$.

\section{Perspectives of patients and caregivers}

The major categories found in patient and caregiver interviews were (1) symptoms and concerns, (2) fear of recurrence, (3) information, (4) needs, (5) satisfaction with care, (6) self-management, and coping (7) preferences for survivorship care planning. Illustrative quotations are presented below and additional quotations are stated in Table 2.

Symptoms and concerns Patient symptoms causing distress were described by the majority of both patients and caregivers (10/13 patients; 9/9 caregivers). Pain (4/13 patients; $2 / 9$ caregivers), neuropathy ( $3 / 13$ patients; $2 / 9$ caregivers), fatigue (3/13 patients, $1 / 9$ caregivers) and anxiety/ depression (2/13 patients; $4 / 9$ caregivers) were the most commonly discussed distressing symptoms. One patient noted that managing her symptoms after treatment ended was particularly challenging:

Post-treatment... that was the hardest time during the whole process because there were a multitude of sideeffects that I was dealing with that ... I didn't have enough information about ...I just wasn't reassured enough that it was going to get better. Or maybe I was unable to absorb that it was going to get better. (Patient 11 , vulvar cancer, stage unknown). 
Table 1 Patient and caregiver characteristics

\begin{tabular}{|c|c|c|c|c|c|c|}
\hline & \multicolumn{3}{|l|}{ Patients } & \multicolumn{3}{|l|}{ Caregivers } \\
\hline & $\begin{array}{l}\text { Total } \\
(N=13)\end{array}$ & $\begin{array}{l}\text { Ovarian cancer } \\
(n=6)\end{array}$ & $\begin{array}{l}\text { Non-ovarian } \\
\text { cancer }(n=7)\end{array}$ & $\begin{array}{l}\text { Total } \\
(N=9)\end{array}$ & $\begin{array}{l}\text { Ovarian cancer } \\
(n=5)\end{array}$ & $\begin{array}{l}\text { Non-ovarian cancer } \\
(n=4)\end{array}$ \\
\hline Age, $M$ (min-max) & $63.1(48-71)$ & $63.0(48-71)$ & $61.7(51-75)$ & $58.7(34-73)$ & $60.4(34-73)$ & $56.5(37-79)$ \\
\hline \multicolumn{7}{|l|}{ Sex } \\
\hline Male & $0(0)$ & $0(0)$ & $0(0)$ & $7(78)$ & $4(80)$ & $3(75)$ \\
\hline Female & $100(100)$ & $100(100)$ & $100(100)$ & $2(22)$ & $1(20)$ & $1(25)$ \\
\hline \multicolumn{7}{|l|}{ Ethnicity } \\
\hline White & $12(92)$ & $6(100)$ & $6(86)$ & $100(100)$ & $100(100)$ & $100(100)$ \\
\hline Asian & $1(8)$ & $0(0)$ & $1(14)$ & $0(0)$ & $0(0)$ & $0(0)$ \\
\hline \multicolumn{7}{|l|}{ Educational level $^{\mathrm{a}}$} \\
\hline $\begin{array}{l}\text { High school diploma or 2-year/ } \\
\text { associate's degree }\end{array}$ & $3(23)$ & $2(33)$ & $1(14)$ & $1(11)$ & $1(20)$ & $0(0)$ \\
\hline 4-year/ bachelor's degree & $3(23)$ & $1(17)$ & $2(28)$ & $3(33)$ & $1(20)$ & $1(25)$ \\
\hline Graduate/professional degree & $4(31)$ & $3(50)$ & $1(14)$ & $3(33)$ & $3(60)$ & $1(25)$ \\
\hline Unknown & $3(23)$ & $0(0)$ & $3(43)$ & $2(22)$ & $0(0)$ & $2(50)$ \\
\hline \multicolumn{7}{|l|}{ Currently employed } \\
\hline Yes & $3(23)$ & $1(17)$ & $2(28)$ & $5(56)$ & $2(40)$ & $1(25)$ \\
\hline No & $8(62)$ & $5(83)$ & $3(43)$ & $2(22)$ & $3(60)$ & $1(25)$ \\
\hline Unknown & $2(15)$ & $0(0)$ & $2(28)$ & $2(22)$ & $0(0)$ & $2(50)$ \\
\hline \multicolumn{7}{|l|}{ Patient clinical characteristics } \\
\hline \multicolumn{7}{|l|}{ Cancer type, $N(\%)$} \\
\hline Ovarian & $6(46)$ & $6(100)$ & N/A & $7(55)$ & $5(100)$ & N/A \\
\hline Endometrial & $4(31)$ & N/A & $4(57)$ & $1(11)$ & N/A & $1(25)$ \\
\hline Cervical & $2(15)$ & N/A & $2(29)$ & $2(22)$ & N/A & $2(50)$ \\
\hline Vulvar & $1(8)$ & N/A & $1(14)$ & $1(11)$ & N/A & $1(25)$ \\
\hline \multicolumn{7}{|l|}{ Cancer stage, $N(\%)$} \\
\hline I & $3(23)$ & $0(0)$ & $3(23)$ & $0(0)$ & $0(0)$ & $0(0)$ \\
\hline II & $4(31)$ & $2(33)$ & $2(28)$ & $4(44)$ & $2(40)$ & $2(50)$ \\
\hline III & $2(15)$ & $3(50)$ & $1(14)$ & $1(11)$ & $2(60)$ & $0(0)$ \\
\hline IV & $3(23)$ & $1(17)$ & $2(28)$ & $3(33)$ & $1(20)$ & $1(25)$ \\
\hline Unstaged & $1(8)$ & $0(0)$ & $1(14)$ & $1(11)$ & $0(0)$ & $1(25)$ \\
\hline \multicolumn{7}{|l|}{ Treatment type, $N(\%)$} \\
\hline Surgery only & $2(15)$ & $0(0)$ & $2(28)$ & $0(0)$ & $0(0)$ & $0(0)$ \\
\hline Chemotherapy & $6(46)$ & $1(83)$ & $1(17)$ & $5(55)$ & $4(80)$ & $1(25)$ \\
\hline Chemo + radiotherapy & $5(38)$ & $1(17)$ & $4(57)$ & $4(44)$ & $1(20)$ & $3(75)$ \\
\hline Months since end of treatment, $M$ (min-max) & $6.4(1-11)$ & $5.2(1-10)$ & $7.4(2-11)$ & $5.9(1-11)$ & $8.0(2-9)$ & $4.2(1-10)$ \\
\hline
\end{tabular}

N/A not available

Almost half of patients (6/13) expressed that they had limited or no post-treatment symptoms, indicating that whatever symptoms they did experience were not distressing.

While some caregivers reported a similar desire for reassurance that physical symptoms would subside post-treatment, they reported many more concerns about dealing with the emotional adjustment. For example, one caregiver described his lack of training in medical symptoms and concern about his ability to emotionally support his wife:
I'm not too concerned with the physical stuff. I can deal with that. Her feet don't work. Her hands don't work. And she's had a profound hearing loss. I have no training background or anything in how to assist with that. But she'll say, 'Can you open this for me?' ... those easy things.... I'm more concerned with the emotional support and maybe being sensitive, those types of things. (Caregiver 7, Fallopian tube cancer, stage IIB). 


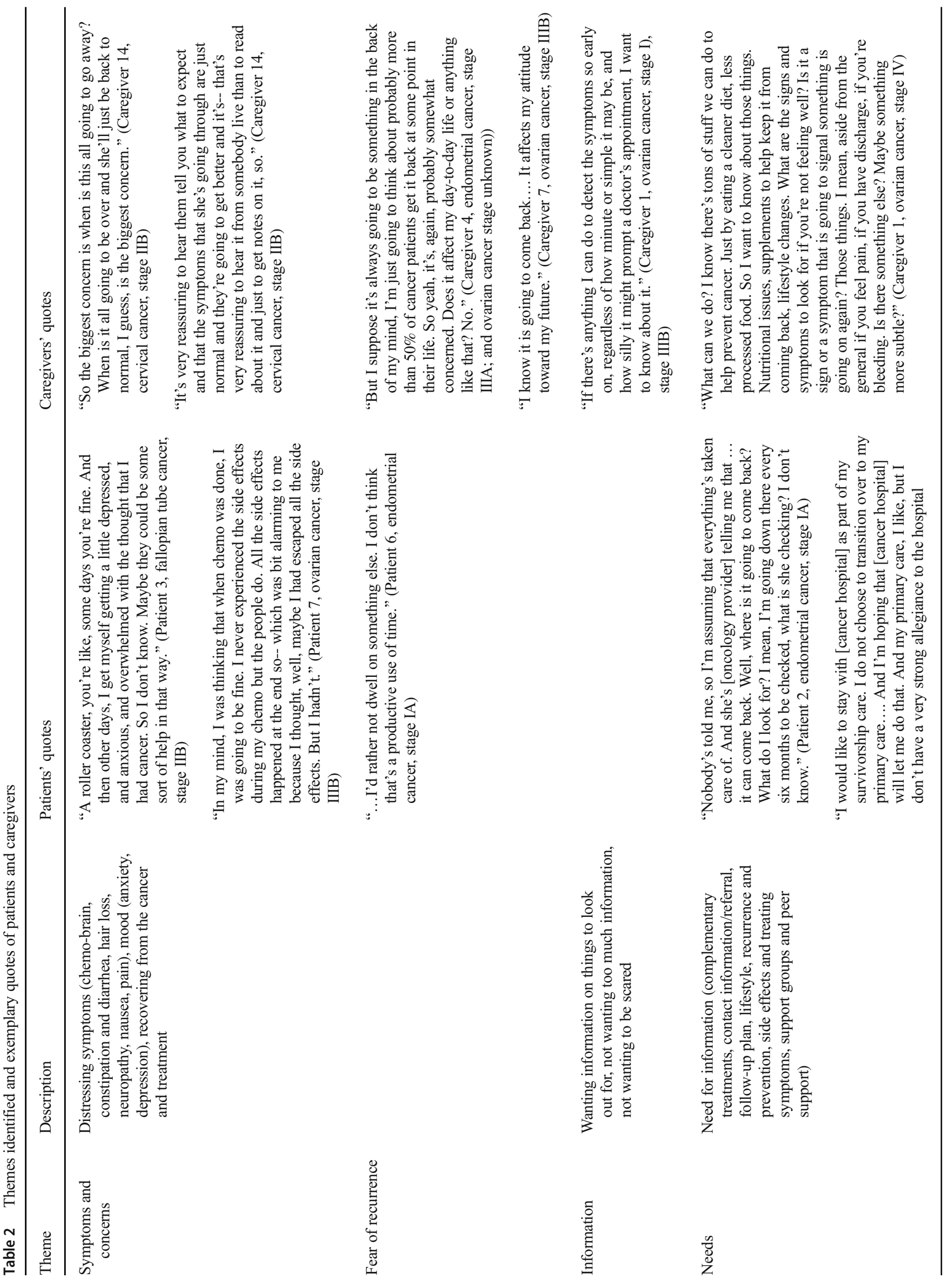




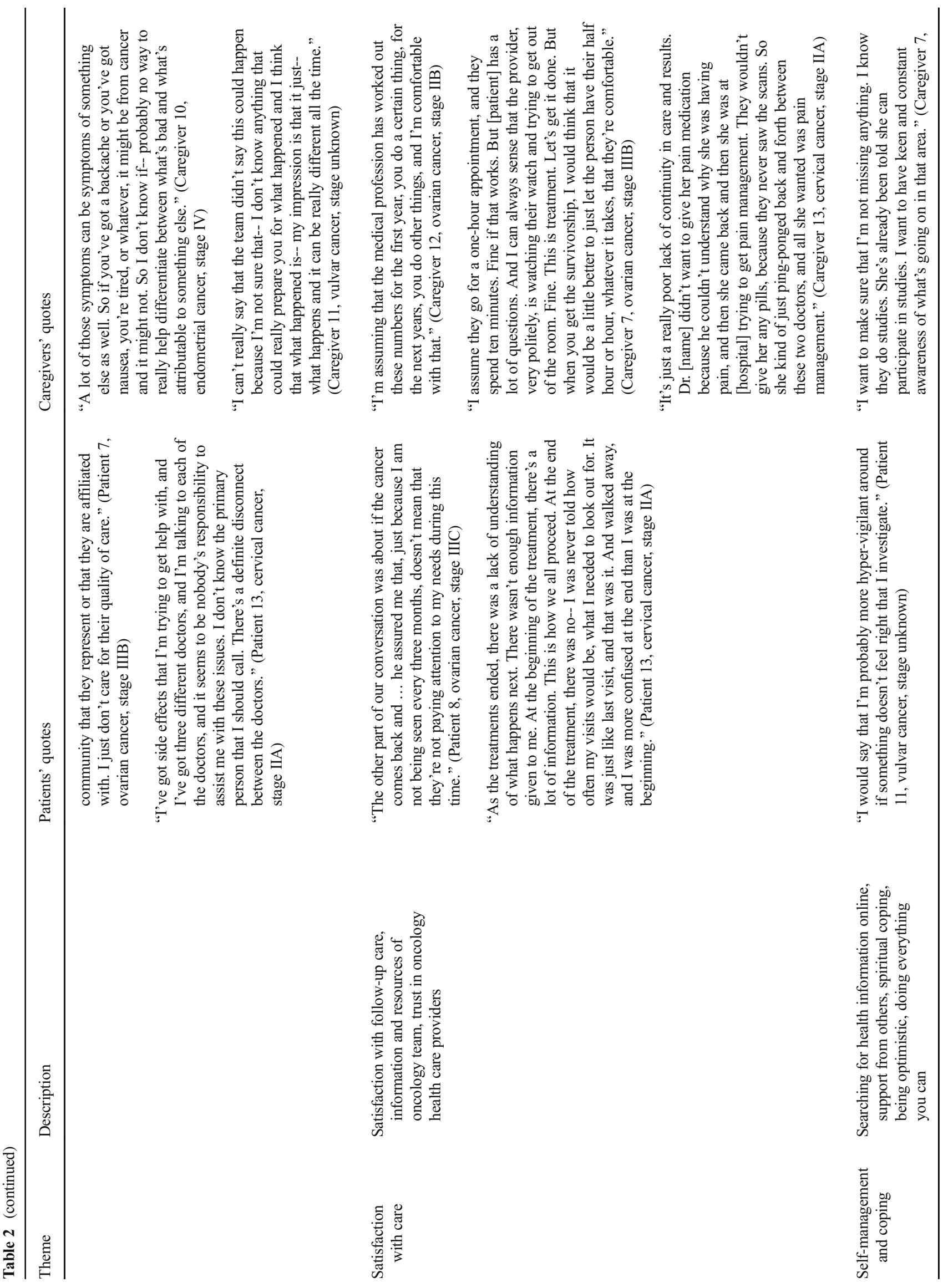




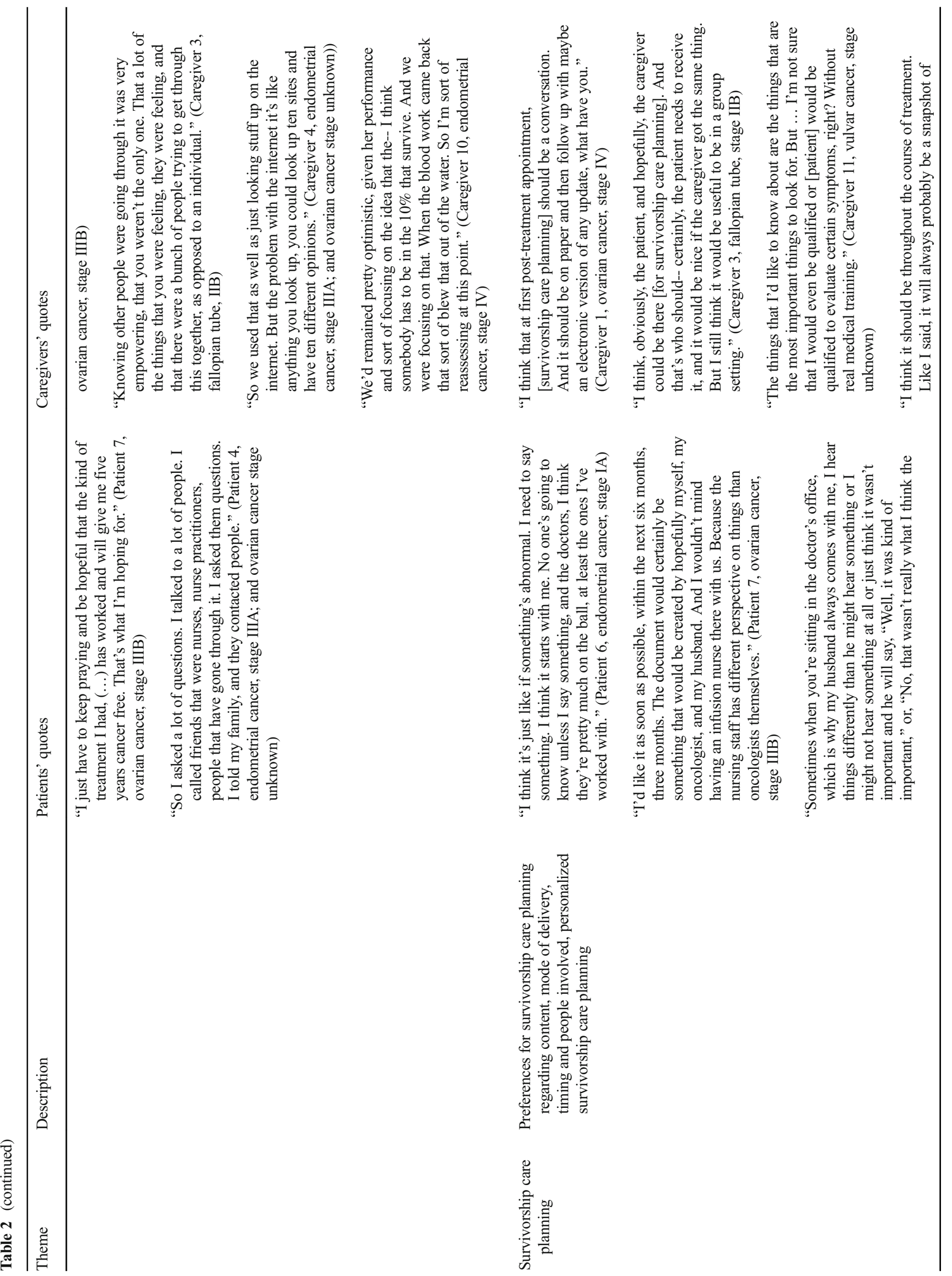


Fear of recurrence Fear of recurrence was common among both patients and caregivers (9/13 patients; 8/9 caregivers). Some patients reported overwhelming preoccupation with the chance that their cancer could return:

I've had a lot of anxiety over it. Like if I get a pain, right away, my head goes to the worst-case scenario. So the fact that I had the cancer, it makes me more anxious about thinking that I could get it somewhere else. (Patient 3, fallopian tube cancer, stage IIB).

Of interest, despite the majority confirming they experienced fear of recurrence, most also noted they were not preoccupied with this fear (10/13 patients; $2 / 9$ caregivers).

Informational needs Informational needs were reported by both caregivers and patients and included possible signs or symptoms of recurrence (11/13 patients; $8 / 9$ caregivers), management of side effects (7/13 patients; $6 / 9$ caregivers), contact information for care providers or sources of specialized services (6/13 patients; $2 / 9$ caregivers), symptom management (4/13 patients, 4/9 caregivers), and methods to reduce risk of recurrence or new cancers (no patients; 5/ 9 caregivers).

Self-management and coping Patients and caregivers wanted to know what the range of expected ongoing issues might be so that they could make informed decisions about when to contact their oncology HCP. Patients saw this as a way to self-manage and control their health:

I am the best steward for my body. I'm the one that looks at it and feels it every day....I need to have as much education as I can have so that I can take care of my body (Patient 8, ovarian cancer, stage IIIC).

Caregivers felt similar desires:

That would be my job to decide or not. But no, I don't want the medical providers deciding that. I want to know everything (Caregiver 7, ovarian cancer, stage IIIB).

Some patients did not want to be scared by the post-treatment side effects:

I don't like to get more information than what I really need to know. I don't want to scare myself.... I was going through this with just being calm and see what happens (Patient 2, endometrial cancer, IA). 
Satisfaction with care Although the majority reported satisfaction with the current informational resources they received from their oncology HCP (11/13 patients; 9/9 caregivers), almost all (12/13 patients; 9/9 caregivers) expressed a need for supplemental information to address their remaining issues and ongoing concerns. Most patients and caregivers reported feeling like they could contact their oncology HCP whenever they needed help:

I'm not that concerned because I know that if something comes up and I'm unsure, I can call them and see them, or I can call them and ask them (Patient 4, endometrial cancer, stage IIIA; and ovarian cancer, stage unknown).

Survivorship care planning Patients and caregivers mainly prefered to receive an SCP in written form (8/13 patients; $5 / 9$ caregivers) though the majority noted that both written and online were acceptable (7/13 patients; 6/9 caregivers). Most wanted the SCP to be updated overtime (9/13 patients; 6/9 caregivers), and many wanted to receive the SCP at first follow-up visit (5/13 patients; 5/9 caregivers). Some did not think that a SCP would be applicable to them (3/13 patients; $1 / 9 \%$ caregivers) because they received minimal treatment:

...They were very thorough with telling me everything that happened. Maybe it might have been different if I was getting further treatment like the chemo or radiation. I think you would want to know more information about that and how this is going to work or, I don't really know. (Patient 2, endometrial cancer, stage IA).

While patients and caregivers varied in their preferences for the ideal content and timing of SCPs, most described their choices as based on their evolving state of health. Therefore, single review of treatment and care plan at the completion of initial therapy as a one time SCP to address survivorship concerns does not appear to be sufficient. Patients and caregivers wanted information when it would be immediately relevant to their health and well-being at multiple points across the disease trajectory:

It all depends on my state of health. If I am very sick, I don't think I even need the information, but if I'm starting with symptoms, as soon as possible. So we, myself and my care team, will have that plan in motion for treatment. (Patient 1, ovarian cancer, stage IV).

\section{Ovarian versus non-ovarian cancer}

Compared to patients with non-ovarian cancer types $(n=7)$, patients with ovarian cancer $(n=6)$ more often reported mood problems such as anxiety and depression (2/6 ovarian; 0/7 non-ovarian) and chemo-brain (2/6 ovarian; 0/7 non-ovarian), while non-ovarian cancer patients more often reported having no or limited symptoms (2/6 ovarian; 4/7 non-ovarian). Coping strategies of ovarian cancer patients were more often spiritual (4/6 ovarian; 1/7 non-ovarian) and trying to be optimistic (4/6 ovarian; $1 / 7$ non-ovarian):

I don't look back. Right now, I don't have cancer and I choose not to think that it's coming back. I'm very positive. I live for today and that's how I manage. I don't know about anybody else but that's my attitude. (Patient 1, ovarian cancer, stage IV).

With regard to survivorship care planning, both ovarian cancer patients and caregivers preferred to receive written information (6/6 ovarian patients; $2 / 7$ non-ovarian patients; $4 / 5$ ovarian caregivers; $1 / 4$ non-ovarian caregivers). Many of the patients with other types of gynecological cancers - but none of the patients with ovarian cancer-reported that they were not interested in a SCP because it was not relevant to their situation (0/6 ovarian; $3 / 7$ non-ovarian).

I think this question [about SCPs] is more for people that have been through a lot more than what I have been through. (Patient 2, endometrial cancer, Stage IA).

\section{Perspectives of health care providers}

The HCP focus group included a detailed discussion on the challenges they encounter while communicating about survivorship. Illustrative quotations are presented below and additional quotes are stated in Table 3. A major barrier to communication was feeling an underlying tension between being direct about the likelihood of a recurrence without stripping away the patient's ability to enjoy life. They reported a reluctance to "scare" patients with information about recurrence and ongoing health issues as a way to help patients focus on enhancing their quality of life:

There's always this really inherent tension in that visit, between stating that [the cancer is incurable] again, and taking away the reprieve that they're about to have.... The tension between being honest and being cruel, or being misleading. And it's very complex, and the language is very complex.... So it's a tight dance (Provider 4, gynecologic oncologist). 
Table 3 Themes identified and exemplary quotes of health care providers

\begin{tabular}{|c|c|c|}
\hline Theme & Description & Health care providers' quotes \\
\hline $\begin{array}{l}\text { Challenges in post- } \\
\text { treatment care }\end{array}$ & $\begin{array}{l}\text { Challenges in communicating about } \\
\text { survivorship, struggling to find necessary } \\
\text { resources for patients, uncertainty about } \\
\text { recurrence, not wanting to scare patients/ } \\
\text { improve quality of life }\end{array}$ & $\begin{array}{l}\text { "I think I use it as a sort of metric about my degree of burnout. If } \\
\text { I'm looking at the end-game for them, and they're depressed } \\
\text { about the potentially bad outcome, I feel like I'm a bit more } \\
\text { burned-out. Whereas, if I'm celebrating with them now, I'm-- } \\
\text { sort of feel like I understand the big picture, but where are } \\
\text { they at now." (Provider 2, medical oncologist) } \\
\text { "Helping people be able to use that good time that they have } \\
\text { because I know that ovarian cancer patients actually spend a } \\
\text { lot of that time just worrying and freaking out. And if care } \\
\text { plan can help with that, but I'd be hopeful it might. Whereas } \\
\text { for early stage in endometrial cancer patients, who we tell, } \\
\text { 'You're probably cured,' I would actually want the care plan } \\
\text { to be a little bit different and I actually want more there to be, } \\
\text { like 'If you have X kind of symptoms, or bleeding, or } \\
\text { whatever, please give us a call.' So I think, to me, I'd want } \\
\text { them to be really shaped to what the general trajectory is that } \\
\text { diseases tend to be." (Provider 7, gynecologic oncology } \\
\text { fellow) }\end{array}$ \\
\hline $\begin{array}{l}\text { Need for survivorship } \\
\text { care plan and resources }\end{array}$ & $\begin{array}{l}\text { Need for SCP (most common issues/percentages, } \\
\text { reassurance, sexual health, support groups, } \\
\text { diet, exercise, attitude, how to get back to } \\
\text { normal, what to expect, follow-up plan), } \\
\text { written information as supplement to } \\
\text { conversation, referencing what to look out } \\
\text { for and when to come back, disease-specific } \\
\text { SCPs }\end{array}$ & $\begin{array}{l}\text { "I don't think I call it the survivorship plan. I think we just come } \\
\text { up with a strategy for how they're going to move forward } \\
\text { with or without their cancer. And we talk about what's sort of } \\
\text { important." (Provider 1, gynecologic oncologist) } \\
\text { "[Patients] seem to think that they're the only ones going } \\
\text { through this process and they feel alone. And I never knew } \\
\text { any of the resources to hook them up with. Like are there } \\
\text { support groups out there? What are the resources they have so } \\
\text { they don't feel so alone and can go forward in the } \\
\text { survivorship period of their lives." (Provider 5, gynecologic } \\
\text { oncology fellow) } \\
\text { "if we had more of a standardized thing that we knew, oh, } 80 \% \\
\text { of people have this, da, da, da, da, and you could kind of run } \\
\text { through that check off and then have the immediate thing that } \\
\text { they needed to get plugged into." (Provider 1, gynecologic } \\
\text { oncologist) }\end{array}$ \\
\hline $\begin{array}{l}\text { Barriers to providing } \\
\text { survivorship care } \\
\text { plans }\end{array}$ & $\begin{array}{l}\text { Barrier of time, not wanting to open up difficult } \\
\text { needs, who should provide SCPs (oncologist, } \\
\text { nurse, anybody), logistics of providing SCPs, } \\
\text { standardized list of prompts/screening tools }\end{array}$ & $\begin{array}{l}\text { "What if you ask somebody and they go to pieces in front of you, } \\
\text { and then you have like a whole new thing and you don't have } \\
\text { the ability to unpack it for an hour and a half. It's really hard. } \\
\text { So how do you do that? And how do you make them feel like } \\
\text { you've heard them?" (Provider 1, gynecologic oncologist) }\end{array}$ \\
\hline
\end{tabular}

Another challenge to communication was prognosis. For patients who were likely to experience a recurrence (e.g., patients with ovarian cancer), providers desired to reinforce that patients should live life in spite of fear and uncertainty:

It's a question of how do we convey to patients that the time that they have in remission is precious and important? And they shouldn't delay life events thinking that they're going to have a really long time to sort of get to that later (Provider 1, gynecologic oncologist).

Despite the information available, HCPs felt they continued to struggle to find necessary resources for patients. They expressed the need for survivorship-care resources to facilitate and support conversations about what to expect after treatment including a follow-up plan. The examples described by one provider describe the extent and details of the resources providers wanted to provide their patients:

I think it would be nice just to have resources about how to get back to your normal life. So what to do if you're depressed or anxious, or how to get sexual function back, or interest, or exercise. So, things not just about the cancer, but how can we get back to your life and living with the cancer (Provider 6, gynecologic oncology fellow).

Providers also expressed that they want tailored and diseasespecific SCPs to assist with difficult conversations, particularly referring to ovarian cancer as being different from other gynecological cancers: 
Because you want to celebrate the win and not tell them that we're going to run out of runway (Provider 1, gynecologic oncologist).

However, the main barrier to providing a SCP to patients identified by providers was lack of time. In addition to concerns over time to develop and present a SCP, some worried that providing a SCP might identify needs or open up conversations that providers could not address during the visit. Gynecologic oncologists preferred to have a medical doctor or other member of the gynecological cancer team provide an SCP, but some felt that this could be provided by a dedicated survivorship specialist as opposed to no one providing SCPs.

\section{Discussion}

This study reports participants' self-identified concerns and preferences for survivorship care. Findings indicate that patients with a gynecological cancer and their caregivers have needs and ongoing issues after treatment, such as side effects and psychological distress, and that they desire information on how to better address these needs. Preferences for survivorship care largely differ across cancer types and individuals, with respect to content, timing, and mode of delivery and reflect the need for disease-specific, tailored SCPs and follow-up care to support care to the diverse group of gynecological cancer survivors. Our results contribute to the ongoing discussion about effective and efficient means to support survivorship care planning in gynecologic oncology, further highlighting the fact that "one size fits all" approaches are unlikely to be successful, and individualized assessment and care planning is needed.

Issues, concerns, and symptoms most often discussed in our study are similar to previous work and include pain, neuropathy, fatigue, and mood problems such as anxiety, depression, and fear of recurrence [2-7]. As reported in previous literature, ovarian cancer patients more often described mood problems and fear of recurrence or progression compared to non-ovarian cancer patients [20]. As a result, compared to non-ovarian cancer patients, ovarian cancer patients more often expressed a need for contact information or referral for someone to help with these concerns.

Caregivers in our study reported similar perspectives as patients, but with several notable exceptions including more frequent endorsement of being afraid of a cancer recurrence or disease progression and wanting to learn health promotion strategies. These results complement growing literature describing the changing and often increasing needs of caregivers of individuals with gynecological cancer [21, 22]. For example, Stafford and Judd found that caregivers' unmet needs were a key predictor of their anxiety, depression, and relationship satisfaction [23]. Integrating caregivers' ongoing unmet needs such as those identified in our study into survivorship care can address their concerns and prevent these negative outcomes. Addressing the concerns and needs of caregivers as an aspect of survivorship care may reduce distress among patients and improve quality of life.

In spite of most patients and caregivers in our study being highly satisfied with information supplied by and resources identified by their HCPs, they still reported informational needs that remained unaddressed. Notably, some stakeholders wish to receive a written document including information about what to expect after treatment and extensive and up-to-date information on specific topics, which largely resembles a Survivorship Care Plan (SCP) as was proposed by the NAM since 2006 [11]. However, other patients and caregivers did not describe a clear need for additional resources or desire for more information. In this wide range of needs and preferences, a "one size fits all" approach may not be most effective nor efficient. This might explain why previous trials assessing the effectiveness of SCPs failed to identify benefits in unselected populations, including samples of American [13] and Dutch [16, 17] gynecological cancer patients. Though women in the latter trial only included endometrial and ovarian cancer, previous analyses suggest that patients' benefit of SCPs is indeed heterogenous [24, 25]. Ideally, survivorship resources should be allocated to those with highest neccesity and be updated over time. This highlights a need for screening for informational and other needs as an important part of survivorship care, and a necessary step in the development of individualized SCPs. Future SCP effectiveness trials should focus on individualized SCPs, particularly when assessed in heterogenous patient populations such as in gynecological oncology.

As most patients and caregivers did not indicate a clear preference for either written or online SCPs, an online, patientcentered application including tailored information for those with specific needs could be a solution that fits the needs of all stakeholders. A written leaflet including more general information could complement the online tool, or even replace it for those with minimal information needs. Further, patients and caregivers who were interested in an SCP indicated that they would like to receive one during the first follow-up visit after the end of treatment, and prefer a conversation accompanied with it, as opposed to generation of a document alone. An important finding of this study is that patients and caregivers do not indicate a strong preference for the person leading this conversation. Conversely, HCPs in our study believe that patients prefer their treating oncologist to provide survivorship care planning. However, they also recognize that this may not be feasible in their practice due to increasing clinical burdens and lack of time. Previous studies also found that lack of time was cited as the greatest barrier to implementation of SCPs [26, 27]. While oncologists buy into the concepts of survivorship 
care planning, the suggestions from providers in our study offer potential ways to address systematic implemention including personalization of care plans to individual patients, inclusion of a dedicated support staff to facilitate discussions, and creation of a prompt list to initiate the discussion using careful but clear communication strategies. Our study supports that patients and caregivers may be amenable to receive SCPs by other members of the care team besides the oncologist, depending on the clinical practices' logistics and feasibility.

This study includes a variety of gynecological cancer types and stages, caregiver types, and gynecologic oncology HCP. Even though our sample was reasonably heterogenous, we reached data saturation for all groups. Our qualitative data allowed for assessment of unique individual and heterogenous experiences of stakeholders. Our findings provide detailed indepth descriptions of the various perspectives in this field and enrich the limited literature available. However, a limitation of this study includes the use of a single medical center to recruit participants, serving patients with a relatively high socioeconomic status and few ethnic minorities. Further, only one patient and her caregiver were clearly dissatisfied with care at our center, which is not in coherence with literature showing much higher proportions of dissatisfaction with care [28, 29], resulting in potentially biased descriptions of concerns and preferences.

\section{Conclusion}

In conclusion, patients and caregivers in this study endorsed the need for personalized, tailored survivorship care planning starting near the end of treatment. Patients with ovarian cancer reported qualitatively different experiences and desires as patient with non-ovarian gynecological cancers, indicating these groups may require distinct forms of care planning. HCPs require assistance in starting sensitive conversations at the end of treatment, but are open to providing individualized SCPs to their patients within the context of the entire team. These qualitative findings provide a description of the self-reported needs of multiple stakeholders, highlight barriers and opportunties to address survivorship needs within the gynecology oncology clinic, and can be used to support the development of patientcentered survivorship care planning interventions.

Funding This work was supported by institutional funds from the Massachusetts General Hospital Cancer Survivorship Program.

\section{Compliance with ethical standards}

The study protocol was approved by the Dana-Farber/Harvard Cancer Center Institutional Review Board. All procedures performed in this study were in accordance with the ethical standards of the institutional and/or national research committee and with the 1964 Helsinki declaration and its later amendments or comparable ethical standards.
Informed consent Informed consent was obtained from all individual participants included in the study.

Conflict of interest The authors declare that they have no conflict of interest.

Open Access This article is distributed under the terms of the Creative Commons Attribution 4.0 International License (http:// creativecommons.org/licenses/by/4.0/), which permits unrestricted use, distribution, and reproduction in any medium, provided you give appropriate credit to the original author(s) and the source, provide a link to the Creative Commons license, and indicate if changes were made.

\section{References}

1. Siegel RL, Miller KD, Jemal A. Cancer statistics, 2017. CA Cancer J Clin. 2017;67(1):7-30.

2. Donovan HS, Hartenbach EM, Method MW. Patient-provider communication and perceived control for women experiencing multiple symptoms associated with ovarian cancer. Gynecol Oncol. 2005;99(2):404-11. https://doi.org/10.1016/j.ygyno.2005.06.062.

3. Ferrell B, Smith S, Cullinane C, Melancon C. Symptom concerns of women with ovarian cancer. J Pain Symptom Manag. 2003;25(6): 528-38.

4. Bradley S, Rose S, Lutgendorf S, Costanzo E, Anderson B. Quality of life and mental health in cervical and endometrial cancer survivors. Gynecol Oncol. 2006;100(3):479-86.

5. Greimel ER, Winter R, Kapp KS, Haas J. Quality of life and sexual functioning after cervical cancer treatment: a long-term follow-up study. Psycho-Oncology. 2009;18(5):476-82.

6. Harrington CB, Hansen JA, Moskowitz M, Todd BL, Feuerstein M. It's not over when it's over: long-term symptoms in cancer survivors - a systematic review. The International Journal of Psychiatry in Medicine. 2010;40(2):163-81.

7. Abbott-Anderson K, Kwekkeboom KL. A systematic review of sexual concerns reported by gynecological cancer survivors. Gynecol Oncol. 2012;124(3):477-89.

8. Matulonis UA, Kornblith A, Lee H, Bryan J, Gibson C, Wells C, et al. Long-term adjustment of early-stage ovarian cancer survivors. Int J Gynecol Cancer. 2008;18(6):1183-93. https://doi.org/10.1111/ j.1525-1438.2007.01167.x.

9. Mirabeau-Beale KL, Kornblith AB, Penson RT, Lee H, Goodman A, Campos SM, et al. Comparison of the quality of life of early and advanced stage ovarian cancer survivors. Gynecol Oncol. 2009;114(2):353-9. https://doi.org/10.1016/j.ygyno.2009.05.009.

10. Ozga M, Aghajanian C, Myers-Virtue S, McDonnell G, Jhanwar S, Hichenberg S, et al. A systematic review of ovarian cancer and fear of recurrence. Palliat Support Care. 2015;13(6):1771-80. https:// doi.org/10.1017/S1478951515000127.

11. Hewitt M, Greenfield S, Stovall E. From cancer patient to cancer survivor: lost in translation. Committee on Cancer Survivorship: Improving Quality Care and Quality of Life, National Cancer Policy Board. Washington, DC: National Academies Press; 2006.

12. Boekhout AH, Maunsell E, Pond GR, Julian JA, Coyle D, Levine $\mathrm{MN}$, et al. A survivorship care plan for breast cancer survivors: extended results of a randomized clinical trial. J Cancer Surviv. 2015;9(4):683-91.

13. Brothers BM, Easley A, Salani R, Andersen BL. Do survivorship care plans impact patients' evaluations of care? A randomized evaluation with gynecologic oncology patients. Gynecol Oncol. 2013;129(3):554-8.

14. Grunfeld E, Julian JA, Pond G, Maunsell E, Coyle D, Folkes A, et al. Evaluating survivorship care plans: results of a randomized, 
clinical trial of patients with breast cancer. J Clin Oncol. 2011;29(36):4755-62.

15. Hershman DL, Greenlee H, Awad D, Kalinsky K, Maurer M, Kranwinkel G, et al. Randomized controlled trial of a clinic-based survivorship intervention following adjuvant therapy in breast cancer survivors. Breast Cancer Res Treat. 2013;138(3):795-806.

16. Nicolaije KA, Ezendam NP, Vos MC, Pijnenborg JM, Boll D, Boss EA, et al. Impact of an automatically generated cancer survivorship care plan on patient-reported outcomes in routine clinical practice: longitudinal outcomes of a pragmatic, cluster randomized trial. J Clin Oncol. 2015;33(31):3550-9.

17. de Rooij BH, Ezendam NPM, Nicolaije KAH, Caroline Vos M, Pijnenborg JMA, Boll D, et al. Effects of survivorship care plans on patient reported outcomes in ovarian cancer during 2-year follow-up - the ROGY care trial. Gynecol Oncol. 2017;145(2):31928. https://doi.org/10.1016/j.ygyno.2017.02.041

18. de Rooij BH, Ezendam NP, Nicolaije KA, Vos MC, Pijnenborg JM, Boll D, et al. Effects of survivorship care plans on patient reported outcomes in ovarian cancer during 2-year follow-up - the ROGY Care trial. Gynecol Oncol. 2017;145:319-28.

19. Nekhlyudov L, Ganz PA, Arora NK, Rowland JH. Going beyond being lost in transition: a decade of progress in cancer survivorship. Journal of clinical oncology : official journal of the American Society of Clinical Oncology. 2017;35(18):1978-81. https://doi. org/10.1200/JCO.2016.72.1373.

20. Ozga M, Aghajanian C, Myers-Virtue S, McDonnell G, Jhanwar S, Hichenberg S, et al. A systematic review of ovarian cancer and fear of recurrence. Palliat Support Care. 2015;13(6):1771-80.

21. Petricone-Westwood D, Lebel S. Being a caregiver to patients with ovarian cancer: a scoping review of the literature. Gynecol Oncol. 2016;143(1):184-92.

22. Butow PN, Price MA, Bell ML, Webb PM, Friedlander M, Group AOCS, et al. Caring for women with ovarian cancer in the last year of life: a longitudinal study of caregiver quality of life, distress and unmet needs. Gynecol Oncol. 2014;132(3):690-7.

23. Stafford L, Judd F. Partners of long-term gynaecologic cancer survivors: psychiatric morbidity, psychosexual outcomes and supportive care needs. Gynecol Oncol. 2010;118(3):268-73.

24. de Rooij BH, Ezendam NP, Nicolaije KA, Lodder P, Vos MC, Pijnenborg JM, et al. Survivorship care plans have a negative impact on long-term quality of life and anxiety through more threatening illness perceptions in gynecological cancer patients: the ROGY care trial. Qual Life Res. 2018;27(6):1533-44.

25. Nicolaije KA, Ezendam NP, Pijnenborg JM, Boll D, Vos MC, Kruitwagen RF, et al. Paper-based survivorship care plans may be less helpful for cancer patients who search for disease-related information on the internet: results of the Registrationsystem Oncological Gynecology (ROGY) Care randomized trial. J Med Internet Res. 2016;18(7):e162.

26. Brennan M, Gormally J, Butow P, Boyle F, Spillane A. Survivorship care plans in cancer: a systematic review of care plan outcomes. Br J Cancer. 2014;111(10):1899-908.

27. Mayer DK, Birken SA, Check DK, Chen RC. Summing it up: an integrative review of studies of cancer survivorship care plans (2006-2013). Cancer. 2015;121(7):978-96. https://doi.org/10. 1002/cncr.28884.

28. Nicolaije KA, Husson O, Ezendam NP, Vos MC, Kruitwagen RF, Lybeert ML, et al. Endometrial cancer survivors are unsatisfied with received information about diagnosis, treatment and follow-up: a study from the population-based PROFILES registry. Patient Educ Couns. 2012;88(3):427-35. https://doi.org/10.1016/j.pec.2012.05.002.

29. Jones JM, Ferguson S, Edwards E, Walton T, McCurdy N, Howell D. Experiences of care delivery: endometrial cancer survivors at end of treatment. Gynecol Oncol. 2012;124(3):458-64. 